\title{
CDISC ADaM Analysis Purpose Terminology
}

National Cancer Institute

\section{Source}

National Cancer Institute. CDISC ADaM Analysis Purpose Terminology. NCI Thesaurus.

Code C117745.

Terminology associated with the analysis purpose codelist of the Clinical Data

Interchange Standards Consortium (CDISC) Analysis Data Model (ADaM). 